
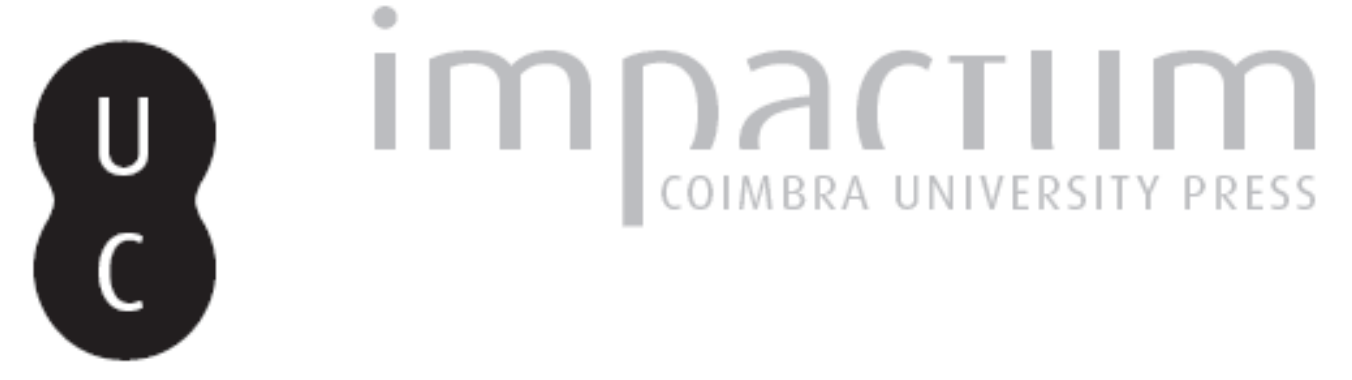

\title{
L'uomo come esistenza che parla: l'orientamento morale e il sentimento religioso di Giovanni da Empoli
}
Autor(es):
Tuccini, Giona

Publicado por: Imprensa da Universidade de Coimbra

URL persistente:

URI:http://hdl.handle.net/10316.2/42580

DOI:

DOI:https://doi.org/10.14195/0870-8584_4_11

Accessed : $\quad$ 26-Apr-2023 12:44:29

A navegação consulta e descarregamento dos títulos inseridos nas Bibliotecas Digitais UC Digitalis, UC Pombalina e UC Impactum, pressupõem a aceitação plena e sem reservas dos Termos e Condições de Uso destas Bibliotecas Digitais, disponíveis em https://digitalis.uc.pt/pt-pt/termos.

Conforme exposto nos referidos Termos e Condições de Uso, o descarregamento de títulos de acesso restrito requer uma licença válida de autorização devendo o utilizador aceder ao(s) documento(s) a partir de um endereço de IP da instituição detentora da supramencionada licença.

Ao utilizador é apenas permitido o descarregamento para uso pessoal, pelo que o emprego do(s) título(s) descarregado(s) para outro fim, designadamente comercial, carece de autorização do respetivo autor ou editor da obra.

Na medida em que todas as obras da UC Digitalis se encontram protegidas pelo Código do Direito de Autor e Direitos Conexos e demais legislação aplicável, toda a cópia, parcial ou total, deste documento, nos casos em que é legalmente admitida, deverá conter ou fazer-se acompanhar por este aviso.

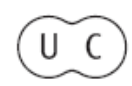




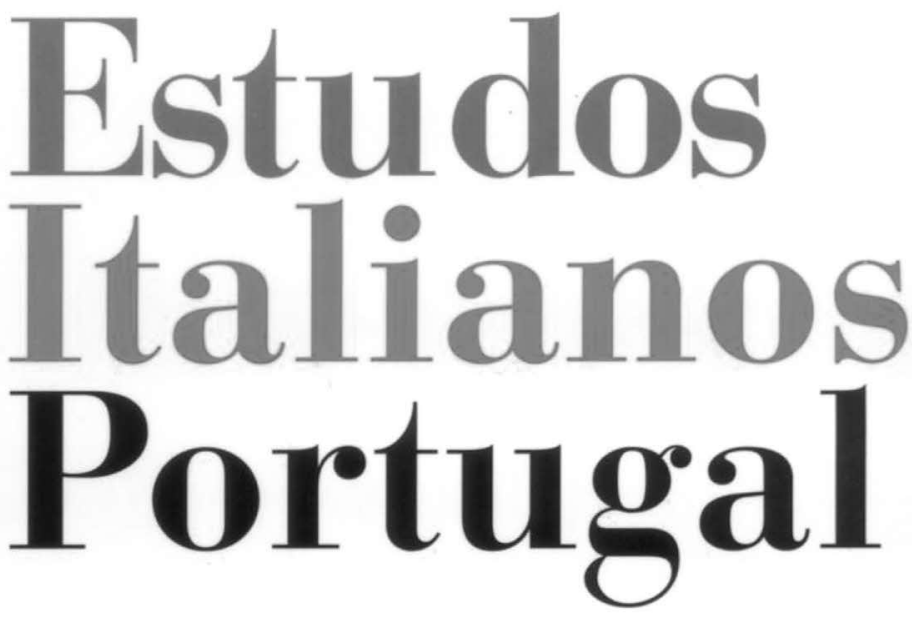

Instituto

Italiano

de Cultura

de Lisboa

Nova Série

$\mathbf{N}^{\mathbf{0}} 4$.

2009 


\section{L'UOMO COME ESISTENZA CHE PARLA. \\ L'ORIENTAMENTO MORALE \\ E IL SENTIMENTO RELIGIOSO \\ DI GIOVANNI DA EMPOLI}

Giona TuCCINI ${ }^{\star}$

All'affettuosa memoria di Luciana S.P., a cui sono debitore per avermi aperto lo scrigno del Portogallo

DALla BIOgrafia SCRITTA amorevolmente dal vecchio zio Girolamo nel $1545^{1}$, veniamo a sapere, dando per buone le informazioni forniteci, che Giovanni da Empoli aveva un'aria assai ben nutrita ed era un bell'uomo: non grasso, non magro, non grande e neanche di piccola statura, di natura sanguigna, allegro, per niente bisbetico, non irascibile ma sempre mite, dotato di ingegno e di una spiccata

* Giona Tuccini (Fucecchio, 1974), laureato in Italianistica all'Università di Firenze, Dottore di Ricerca in Lingue e Culture del Mediterraneo. Si è occupato prevalentemente di letteratura medioevale e rinascimentale, di misticismo e letteratura religiosa, di autori e tendenze del Novecento letterario. Tra le sue pubblicazioni più recenti si ricordano i libri Il vespasiano e l'abito da sposa (Udine, 2003), Spiriti cercanti (Urbino, 2007), Voce del silenzio, luce sul sentiero (Urbino, 2008), l'edizione italiana di J. R. Ackerley, Il mio cane Tulip (Roma, 2007) el'edizione commentata di Bernardino da Siena. Novellette, aneddoti, discorsi volgari (Genova, 2009).

${ }^{1}$ La Vita di esso Giovanni da Empoli da che nacque a che mori, che giaceva inedita nella Magliabechiana, venne pubblicata prima da Giuseppe Canestrini e Filippo Luigi Polidori nella Viola del pensiero. Ricordo pel MDCCCXLII, miscellanea di Letteratura e Morale compilata da Silvio Giannini, a. 3, Livorno, Tipografia Sardi, 1841, pp. 103-132; poi nella citata appendice dell'Archivio Storico Italiano, insieme a lettere di Giovanni da Empoli, illustrate da Gräberg di Hemsö; infine tra le biografie degli Empolesi illustri, raccolte da Alfonso Monti e aggiunte alla Storia di Empoli di Luigi Lazzeri, Empoli, Monti, 1873. Nel 1999 è stata ripubblicata nell'ammirevole volume di Marco Spallanzani, Giovanni da Empoli, un mercante fiorentino nell'Asia portoghese, Firenze, Studio per Edizioni Scelte (S.P.E.S.), 1999, pp. 121-135.

Est.Ital.Port., n.s., 4, 2009: 155-168 
facilità nello studio ${ }^{2}$. Aveva avuto una formazione conforme a quella conosciuta dagli scolari appartenenti al suo stesso ceto sociale: il libro dei Salmi a sette anni, lo studio delle lettere, la Grammatica latina di Donatello (appresa dai maestri di grammatica Girolamo del Maestro Luca, il prete Guasparre Massani, Francesco da Urbino e tale Guerrino), la perfetta padronanza del latino a tredici anni e i primi rudimenti di greco a quattordici. Un bambino, Giovanni, che non avrebbe potuto esimersi dal crescere pio e buono, considerata la famiglia profondamente devota e sentitamente attiva in una Firenze divisa dai contrasti tra i seguaci di Girolamo Savonarola e i fiancheggiatori della Chiesa romana e dei Medici. Fu Leonardo, il padre, a preoccuparsi in prima persona dell'educazione del figlio, nonché a prendersi cura della sua preparazione religiosa: in un libretto agile e maneggevole egli raccolse, per Giovanni, i testi sacri affinché li portasse sempre con sé. E così fece il nostro Sabedor de mar in occasione della sua audace avventura asiatica, per conto di Emanuele I di Portogallo. Con quel libriccino, Leonardo gli rendeva accessibili, in qualsiasi momento, specie nella derelizione e nel dolore, $i$ testi esemplari della tradizione cristiana: i Salmi, le sentenze di Salomone, alcuni passi tratti dal libro della Sapienza e dalle lettere di Paolo; e poi Agostino, Ambrogio e i Vangeli affinché egli ne avesse sempre a mente e perché la pratica di quelle letture fosse un continuo esercizio del vivere nelle cose di Dio. La qualità - e diremo pure la caratteristica - della citazione erudita di Giovanni da Empoli, nelle relazioni odeporiche, trae le sue origini da questo taccuino e, contestualmente, dalle informazioni ricevute durante la propria preparazione scolastica. Indubbiamente, le prediche del Savonarola influenzarono molto il periodo della maturazione del da Empoli che, insieme

2 Girolamo da Empoli, La vita di Giovanni da Empoli, Marco Spallanzani, cit., p. 123. 
ad alcuni ragazzi, tra cui Princivalle della Stufa, prese parte ad attività religiose e sociali volte a denunciare il degrado morale della società del tempo ${ }^{3}$. Il "profeta disarmato" - così Machiavelli amava chiamare il Savonarola - non mancò certamente di trasmettere a quei giovani il messaggio della sua De ruina mundi del 1472, in cui si dichiarava disgustato dalla decadenza dei costumi. Lo stesso Giovanni, come egli ricorda nel suo testamento, pensò di farsi frate nel Convento di San Marco ${ }^{4}$.

Sarà subito compreso, dunque, che negli scritti di viaggio, diligentemente raccolti da Marco Spallanzani qualche anno fa, l'austera formazione domenicana, alla base del pensiero del da Empoli, lascia sempre una traccia suscettibile di manifestazione; a volte sotto forma di citazione biblica, certe altre nei termini di un orientamento morale. Nessuna innocenza valse l'incontro del ragazzo con il frate di Ferrara. Anzi, in compagnia di altri coetanei cresciuti sotto la severa egida del domenicano, il figlio di Leonardo vagava per la città di Firenze requisendo carte e dadi, libri d'amore e novelle sconce, per mandare poi tutto al fuoco: "puossi dire che non fusse mai fanciullo", scrive il biografo che racconta, inoltre, come il nipote ed i suoi compagni andassero a svergognare le dame che circolavano per la strada con lunghi strascichi e sprechi di stoffe ${ }^{5}$. Nel comportamento dell'adolescente c'era un nonsoché di rigido che sfugge alla mentalità giovane: una morigeratezza, una legge, un divieto interiore al quale obbedire. Giovanni aveva la gravità e il silenzio dell'adolescente prematuramente coinvolto in storie d'adulti. Per questo motivo la sua non fu una giovinezza naturale e

3 A tale proposito ritengo esplicativa la lettura di Uzielli Gustavo, "Piero di Andrea Strozzi, viaggiatore fiorentino del secolo delle scoperte", in Memorie della Società Geografica Italiana, 5, 1895, pp. 110-148.

${ }^{4}$ Marco Spallanzani, cit., p. 222: "Io mi botai, come vi schripsi, di farmi frate di San Marcho".

${ }^{5}$ Ib., p. 124. 
innocente ma, piuttosto, una maturità primaticcia verso la quale il ragazzo veniva sospinto dalla cultura dell'epoca che lo sommergeva. Del resto, la testimonianza dello zio ci spiega che a quel tempo il crescere in fretta e con temperanza era, in una famiglia di lavoratori e di timorati di Dio come la loro, il valore supremo della vita.

A questo punto, sarà interessante notare come l'intransigenza e la tendenza a screditare l'Altro che, nell'età della maturazione, pungulavano il da Empoli ad operare in qualità di giudice supremo e denigratore delle vanità fiorentine, residuino in quelle parti delle relazioni in cui egli descrive l'incontro con le popolazioni autocnone, a partire dalle loro peculiarità etniche. In questi scritti, la fisionomia dell'uomo-individuo-viaggiatore si sovrappone a quella dell'uomo-portatore di razza e di cultura, ed è una fisionomia rigida, statica e stabilita una volta per tutte. La visione delle popolazioni locali, che il mercante toscano intende fornirci, è intrisa di demagogia, di disorientamento spacciato per lucidità, di fanatismo. Ciò si spiega perché l'aspetto temibile del nazionalismo - diremo pure della religiosità - di Giovanni da Empoli è il suo essere inseparabilmente associato al disprezzo per il diverso; un disprezzo che non tarderemo a definire "generazionale". Una irrinunciabile componente della concezione del mondo del selvaggio è, non a caso, per lui, la religione ("Fede nessuna non tenghano salvo epichura", scriverà degli indigeni brasiliani, "Li detti gientili sono idolatri" annoterà, invece, parlando dei malabari). La religiosità del viaggiatore, che negli scritti emerge sempre in qualità di un motivo di confronto con l'Altro, si manifesta su un duplice piano: da un lato, su quello di una esplicita fede nell'esistenza e della presenza della trascendenza, di un Essere supremo che è Cristo; dall'altro, su quello della religione in quanto istituzione, forza sociale e perfino politica (qui la tenace memoria del Savonarola, alla base dell'orientamento morale del nostro mercante, gioca un ruolo importante; ma 
anche l'amicizia che, più tardi, stabilirà con i Medici). Non a caso, quando il destino porta Giovanni ad imbattersi in un altro - sia egli un "selvaggio" come gli índios brasiliani o un uomo "evoluto" come gli abitanti del Sud-Est asiatico - egli ne noterà soprattutto tre caratteristiche principali: la razza, il sistema di difesa e la religione. E se gli índios e gli aborigeni africani non rappresentano un interlocutore rispettabile, ma al massimo un oggetto di studio, gli indiani e i cinesi - già solidamente attestati nell'immaginario degli occidentali grazie anche a Marco Polo - vengono considerati un vero partner, corresponsabili cioè della fortuna commerciale della vecchia Europa.

Dobbiamo affermare che l'atteggiamento emergente dagli scritti del da Empoli va incontro al gusto dei tempi. Prendiamo la relazione del primo viaggio, dove si parla della curva a gomito che, nei pressi delle Isole del Capo Verde, la flotta di Emanuele I, prima di guadagnare il Capo di Buona Speranza, percorreva verso l'Isola di Vera Cruz (proprio come faceva Pedro Álvares Cabral, allo scopo di sfruttare venti e correnti favorevoli). Ebbene, in questo scritto, tornano a stupire gli antropofagi (gli stessi che deglutirono Padre Sardinha), ci sono gli índios eroticissimi con la loro bellezza indomita; c'è l'esigenza dell'esploratore di descrivere e di provare a farsi esatto informatore dei costumi, della pratica bellica, della religione del popolo visitato; cultura e modi di essere che vengono sempre esperiti all'insegna di una continua ponderazione morale e approfonditi da un segreto spirito discettivo:

Detta isola non è di nullo di minero [era di nullo valore], per quanto potessimo comprendere [potemo comprendere]; e d'essa partiti, navighando pure in detta volta, ci trovamo tanto avanti chome la terra di Vera Crocie [per mezo la terra di Vera Croce, over del Bresil] così nomata [così nominata], altra volta dischoperta per Amerigo Vespucci, nella quale si fa buona 
somma di cassia e di verzino: altro da minero non abiamo compresa [altro di momento non abbiam compreso]. Le gente d'essa sono di buona forma, vanno nudi, sì huomini chome donne [così uomini come donne], sanza niente coprire [senza coprire niente]; isforacchionsi insino alla cintura [sforacchiansi così in pelle insino alla cintura] e s'adornano di penne varie [verdi] di pappaghalli, e le loro labbra [sono] piene d'ossa di pescie. Le loro arme [sono] come dardi, la punta coperta di dette ossa di pescie. Fede nessuna non tenghano [non hanno] salvo epichura [epicurea]; mangiano per chomune vitto [uso] charne humana [carni umane], la quale [le quali] secchano al fummo, come noi la charne del [di] porcho ${ }^{6}$.

E una volta raggiunta l'Africa Australe - con fatica e travagli, dato il procedere "ad arbor secco senza palmo di vela" - i negroidi di quelle terre gli apparvero repellenti, brutti, quasi teratomorfici:

Gli huomini sono sanza chapegli [senza capelli], e 'l chapo tingnioso [col capo tignoso] e brutto, con gli ochi cispiosi [occhi cispi]; e il chorpo insino [fino] alla cintura [è] vestito di pelle pilose [pelli pelose], e gli huomini portano la loro natura in uno quoio piloso [le loro nature in un cuoio piloso], a modo di ghuaina, sempre diritta. [E] le donne portano detto abito di pelle e a esso apicchato [appiccano] una choda pilosa di dette simile bestie [di simil bestia], le quali pendano dinanzi e drieto [pendono dinanzi e di dietro], per choprire sue verghongnie [coprir le lor vergogne] e ànno [hanno] le poppe loro molto lunghe, cosa molto diforme. Gli huomini portano certi dardi con una punta di ferro, che vi se ne truova qualchuno [che se

${ }^{6}$ Ib., pp. 140-141. Tra parentesi quadre comprendo le porzioni difformi contenute nell'edizione di Giovanni Battista Ramusio, Navigazioni e viaggi, a cura di M. Milanesi, 6 voll., Torino 1978, I, p. 744. Da notare come in questo passo il da Empoli preferisca i termini europei (verzino) per designare oggetti e usanze, senza servirsi delle voci indigene (pau brasil, in questo specifico caso). 
ne trova qualcuno]. Leggie nessuna non tenghano [legge nessuna non tengono] salvo epicurea; mangiano charne cruda, per quanto abiamo veduti [habbiam veduto]; parlano in ghola [e] con cenni e fischi, e giammai e giamai gli abbiamo veduti splichare [esplicar] parola squisita [espedita], perché avamo in fra noi huomini sabitori di varii linghue [avevamo fra noi huomini che sapevano varie lingue], e ggiamai potettano pigliare [potettono pigliar] costrutto di lor linghua: e in conclusione sono huomini bestiali. Questo è quanto s'è compreso in detta terra [abbiam potuto comprendere di detta terra $]^{7}$.

I discorsi di scoperta del mercante fiorentino s'innestano su un codice di scrittura consolidato e inseparabile dalla preoccupazione morale; una morale, espressa personalmente con sentenze, che suggerisce la loquela stessa e che spiega anche la visione stereotipata dei popoli visitati, inducendoci a considerarla - l'abbiamo suggerito sopra - come mero indirizzo di lettura. Le sue descrizioni contengono dettagli di verità che, a causa della visione fanatica e razzista dell' Altro, vengono infarcite di assurdità e di falsi stereotipi. Per questa ragione, ai Tropici e nell'Africa australe l'uomo rinascimentale bianco è la fonte meno degna di fede circa la presentazione delle popolazioni e le culture locali. E non potrebbe essere altrimenti perché egli domina il mondo non solo politicamente ed economicamente, ma anche culturalmente: impone la fede (quella cattolica, s'intende), stabilisce la legge, le scale di valori, i modelli di comportamento, le lingue. La relazione con l'Altro viene sempre sbilanciata in favore dell'occidentale, vale a dire a favore di una interpretazione del "Nuovo mondo" quantomai autoritaria, apodittica e paternalistica. Il verificarsi di uno sbilanciamento così ingiusto e falso ha creato, nei viaggiatori e nei diplomatici del Cinquecento, una serie di consuetudini dure a morire; 
giudizi di valore che, poco fa, ho definito "generazionali". Francesco Massaro, ad esempio, ambasciatore veneziano a Buda nel 1523, mostrava lo stesso spirito del da Empoli in Brasile, denigratorio, sardonico e arrogante, nei confronti degli ungheresi; cittadini che non avevano nulla in comune con gli africani o gli amazzonici, eppure erano "diversi" quanto basta da ispirare un quadretto altrettanto ingiurioso:

Hongari in univerali sono la pegior generation dil mondo. Non amano né extimano natione dil mondo, nepur se amano tra loro. Ogniuno atende al proprio comodo, et robano el publico et poco se curano de quello; hanno uno odio e simultà tra loro occulta che non si potria credere, et tamen alternatim ogni giorno mangiano insieme che pareno fratelli. [...] sono superbi et arroganti e sciano né reger, né gubernar, né voleno consiglio da cui sa $[\ldots]$ assai ben prompti ad determinar, ma ad exeguir tardissimi, et poche cose se mandano ad executione, salvo che il crapular et rubar il publico: a queste cose sono diligentissimi [...] E bisogna esser ben cauti ad negotiar cum loro et, ut multa in unum colligam, hongari sono la fece dil mondo, e se non fosse tanta bontà et innocentia di quel Re, la Divina iustitia non poria tardar tanto alla destrutione de questi hongari ${ }^{8}$.

Ma restiamo al da Empoli. Egli dà prova di non essere alieno dal coltivare gli stessi pensieri e gli stessi pregiudizi dei colonizzatori portoghesi dell'epoca; con loro condivide, addirittura, uno stesso deposito di temi. Lo abbiamo notato con la coppia vistosamente antinonimica índios-negri; antinonimica perché, malgrado vengano considerati entrambi popoli antropofagi e perlopiù nudi, mentre l'índio si presenta agli occhi dell'europeo di una sensualità continuamente insorgente ("sono di buona forma"), senza per altro essere

8 Marino Sanudo, I Diarii, Venezia, Visentini, 1892, t. 35, pp. 108-109 (poi Bologna, Forni, s.d.). 
convertibile, il negro impone al da Empoli un certo sdegno ("cosa molto diforme [...] in conclusione sono huomini bestiali”), nonostante la sua risaputa convertibilità al cristianesimo; qui la sensualità è repressa, eppure non manca di spingere il navigatore - uomo che guarda - a frugare tra le pieghe e tra le malie delle creature selvagge ("le poppe loro molto lunghe"); visioni, quindi, che sembrano affiorare da un abisso angelico e diabolico e che, sulla carta con la parola, diventano ragione di abominio e scherno ("chapo tingnioso [...] ochi cispiosi"). La questione della descrizione dell'Altro avviene, in entrambi i casi, nella dinamica dei suoi comportamenti (l'índio "isforacchionsi insino alla cintura", adornato di penne varie e con le labbra piene "d'ossa di pescie"; il negro "vestito di pelle pilose" con "natura in uno quoio piloso a modo di ghuaina, sempre diritta") e quasi costringe il viaggiatore a dare conto soprattutto dell'aspetto fisico, attraverso l'osservazione visiva quale strumento di conoscenza efficace e apparentemente oggettivo. Potremmo dire, ancora meglio, che il da Empoli riferisce esclusivamente su quanto accertato con i propri occhi ("per quanto abiamo veduto"; "Di che udito e visto"), secondo formule tradizionali, senza intenzione apparente di abbellire o svalutare ("Questo è quanto s'è compreso in detta terra"). In realtà, è subito inteso che la scelta dei tratti fisici è operata esclusivamente in relazione alla "differenza" del soggetto incontrato e fortemente connotata secondo morale: l'esploratore sente l'esigenza di attribuire alla nudità dei valori diversi, distinguendo quella armata da quella ornata, quella naturale da quella "artificiosa", quella innocente e libera dell'indio del Brasile da quella ripugnante e irrimediabilmente negativa dell'africano. Da notare che il concetto di "vergogna" compare soltanto nei passi riferiti a quest'ultimo (il negro viene osservato frontalmente, aggressivamente), mentre nell'altro caso la descrizione risulta più sobria ("vanno nudi, sì huomini chome donne, sanza niente coprire") giacché si tratta di un guardare che è piuttosto un 
ammirare (qui il concetto di "vergogna" si dissolve per lasciare spazio a parole di rispetto). Con queste informazioni il vecchio Mondo - più ridicolo che sinistro - incontrava l'indigeno al vertice della contraddizione, e cioè con la presunzione di ritenerlo l'uomo nuovo ("avamo in fra noi huomini sabitori di varii linghue e ggiamai [i negroidi] potettano pigliare costrutto di lor linghua").

L'esperienza del da Empoli conferma che, di primo acchito, il pioniere reagisce all'Altro con diffidenza, riluttanza, se non addirittura con ostilità, malgrado la celebre ammonizione contenuta nel Levitico: "Ricordati che sei stato straniero!"9. I greci chiamavano bárbaroi i non greci, termine che resta illiquidato nelle sezioni delle reportationes sopracitate, in cui il viaggiatore fiorentino descrive arrogantemente chi non parla la sua lingua, finendo per tenerlo a distanza e per sottometterlo. Lo abbiamo visto. Per un europeo che, in quel tempo, muoveva alla conquista del pianeta, sia geografica che commerciale, l'Altro si configura spesso come un selvaggio nudo, cannibale e pagano che comunica con suoni animaleschi, non con costrutti; un essere riprovevole che l'uomo occidentale - bianco, cristiano, magnificamente loquace - deve assoggettare e calpestare. Bisognerà aspettare l'Illuminismo di Jean-Jacques Rousseau perché i rapporti tra l'Europa e gli Altri si rinnovino a partire dalle fondamenta filosofiche; affinché, cioè, nasca un sentimento inedito e un lessico nuovo per descrivere l'Altro. Solo allora gli enciclopedisti diffondono l'idea di una scienza universale, Goethe sogna la nascita di una letteratura mondiale ed i concetti quali "governo mondiale" e "abitante del mondo" ottengono piena cittadinanza nelle relazioni di viaggio. La parola "selvaggio", più volte impiegata dal da Empoli in senso deteriore, resterà in uso anche nel Settecento, ma verrà corredata dall'aggettivo "buono". 
Se nei discorsi sulle popolazioni sudamericane e africane, il viaggiatore risulta essere, anzitutto, un nazionalista che, come valori supremi, pone la propria cultura, religione e lingua al centro dell'incontro con l'Altro, quando entra in contatto con gli indiani e i cinesi, il mercante si apre ad una nuova dimensione dell'ego: non quella volta a isolare e a stigmatizzare la diversità, bensì quella di un individuo che comprende in sé l'alterità. Dopo le tante "stranezze" registrate tra i popoli del Pau Brasil e dell'Africa Australe, con la paura del "diverso" che evocava figure orrende e risvegliava gli istinti, a Malabar il da Empoli incontra gli indiani, "huomini necti e puliti", con cui egli si trova tanto a proprio agio da non sentire quasi la curiosità di menzionarli al padre, se non nella parte finale della prima relazione:

Schordavami di dirvi e costumi e modi de' Malabari e gientili de l'India, le quali per falta di buona memoria l'avevo dimentachata. Li detti gientili sono idolatri, non maggiono charne, né pesce, né huova, né chosa che tengha sanghue; solo mangiono risi e erbe. Sono huomini necti e puliti; habitano in chase murate di mattoni e chalcina, bene lavorate. Tenghano le vacche per loro dio, sì ne e ve n'è assai abondanza per tutto la terra. Questo è quanto abiamo compreso, ecc., e di tanto vi fo noto, che Iddio vi acrescha vostra vita ${ }^{10}$.

All'esploratore non interessa la descriptio rerum in sé, se non in relazione all'uomo, come c'informano gli ampi spazi riservati alla descrizione delle abitazioni degli indigeni, dei loro possedimenti, dei loro costumi. Nella lettera relativa al secondo viaggio, quello a Malacca, il mercante trova ancora modo di informare il padre circa una popolazione delle Isole Nicobare, nell'Oceano Indiano: i Bantù. Giovanni chiama la gente di questo popolo con lo stesso nome datogli dagli

${ }^{10}$ Marco Spallanzani, cit., p. 151. 
Arabi, ossia "Cafri”" (dall'arabo Käfir, "miscredente", "infedele"), nome con il quale si chiamarono più in generale tutte le popolazioni di colore non islamizzate. Egli si serve di questo termine attingendolo proprio dal portoghese, dacché era passato nell'uso di questa lingua con l'accezione dispregiativa di "barbari"".

$\mathrm{Ma}$, in definitiva, quali sono le sorti e le finalità dello spirito religioso di Giovanni da Empoli, nelle sue relazioni? Sul piano del récit, noteremo subito che tale sentimento suggerisce la formula di apertura e di chiusura di qualsiasi lettera che abbia un destinatario ben preciso. Si tratta di formule convenzionali che delimitano la cornice epistolare vera e propria. Un esempio? La lettera da Malacca al padre: l'inizio è preceduto dalla canonica invocazione a Dio

Onorando padre. La vostra beneditione con quella dello Etterno, sia quella che mmi ghuardi et benedicha ecc.

poi ripristinata alla fine dell'epistola

Che Dio di mal vi ghuardi, et ci dia gratia che presto ci possiamo rivedere ecc...

Sul piano sociale, il discorso si fa un pochino più articolato: Dio non è un motto ma un impresario immaginario. Già nel saggio "Per mare ed oltre. Il punto sui ricordi di viaggio di Giovanni da Empoli (1503-1518)""12, scrivevo della compatibilità dei fini commerciali con quelli religiosi alla base

11 Ib., p. 194.

12 Giona Tuccini, "Per mare ed oltre. Il punto sui ricordi di viaggio di Giovanni da Empoli (1503-1518)”, negli Atti del Convegno Portogallo e Asia - Università degli Studi di Napoli "L'Orientale", 5-6 dicembre 2005 e 3 marzo 2006, a cura di M. L. Cusati, Napoli, Il Torcololiere Officine Grafico Editoriali d'Ateneo, 2008, pp. 145-184 (su supporto CD-ROM), poi sensibilmente variato in Critica del testo, 11, 3, 2008. 
delle attività del da Empoli. Nella lettera indirizzata al padre dall'India (quella raccolta da Ramusio), egli esprimeva con chiarezza la sua convinzione circa tale compatibilità, per cui il navigar mercantesco si svolgeva collateralmente ad un'impresa più propriamente evangelizzante. Leggiamo qui:

Et sine dubio credo che, chon l'aiuto di Dio, non solamente el serenissimo re di Portoghallo arà acquistato gran richeza, ma etiam sono di credere che in ispatio di 50 anni saranno convertiti assai giente, che iddio ne presti di sua infinita gratia ${ }^{13}$.

Durante il viaggio verso la penisola malesiana, varie volte Giovanni ebbe l'incarico di scendere a terra per negoziare con i capi barbari a nome del Re del Portogallo e trattare mercanzie. Ma anche la città di Malacca, come quella di Goa, dovette essere presa di forza. La lettera che il mercante scrisse sulla Malesia a suo padre Leonardo, e che terminò soltanto il 20 luglio 1514 nelle Azzorre, poco prima del suo arrivo a Lisbona (come testimonia la postilla di chiusura), contiene la descrizione dell'assalto ordinato dall'Albuquerque, dopo che tutta la sua gente si fu confessata e comunicata: a un certo punto del racconto, il tono dell'empolese è quello di un crociato che si sente portatore - ancora una volta - di valori universali, dacché il Dio a cui s'appella è lo stesso che dava la vittoria ai Cristiani contro gli infedeli:

Noi eravamo circha 1500 uomini; avamo e Cini in aiuto, che erano circha 400; il principe di Zamatora chon altrettanti: et chosì demo la battagla il giorno dello appostolo Sancto Iacopo, padrone e interciessore di Spagna. E chosì, davanti giorno, dato l'assalto e montati su lo stechato, dove si combatté assai, e ssi difesono gentilmente e massime con elefanti armati, in uno de' quali era il figliuolo del re: facevangli mughiare, che era chosa

${ }^{13}$ Marco Spallanzani, cit., p. 149. 
da spaurire il mondo. Amazamone uno chon l'artigleria, ferimo il principe, faciemo grandissimo uccisione. E chome miracholosamente Nostro Signore sempre dà vittoria a' sua cristiani contro agl'infedeli, sendo nostro capitano l'Apostolo Sa Iacopo, con pocha gente che eravamo, si superò la grandissima moltitudine di nimici, che ssono valentissimi huomini e bene instruiti nella ghuerra, abondanti d'ogni generazione d'arme molto buone. E chosì quello giorno ghuadagnamo la cipttà, e rubamola in molta parte ${ }^{14}$.

Da questo brano si evince che, diversamente da quanto ha creduto Domenico da Empoli in "Religiosità e spirito mercantile in Giovanni da Empoli"15, le speranze del viaggiatore in Asia di vedere diffusa la fede cattolica, nel corso delle spedizioni portoghesi, non erano destinate a cadere come illusione giovanile, ma a permanere nell'uomo adulto come il migliore dei propositi.

14 Ib., p. 186.

15 Domenico da Empoli, "Religiosità e spirito mercantile in Giovanni da Empoli", in AA.VV., Miscellanea di storia delle esplorazioni, vol. 33, 12, Genova 1987, p. 12. 